Military Technical College, Kobry El-Kobbah, Cairo, Egypt

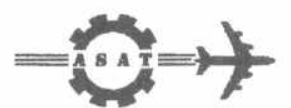

$9^{\text {th }}$ International Conference

On Aerospace Sciences \& Aviation Technology

\title{
FAR FIELD SCATTERING OF GAUSSIAN BEAM BY AN INFINITELY LONG CONDUCTING CIRCULAR CYLINDER
}

\author{
AZZAM A. M. " BADR Y. A. "ALY M. S. " hasan M.F. " ElmahdY A. M. "
}

\section{Abstract}

The Gaussian beam scattering from an infinitely long conducting circular cylinder with large size and located in the far zone from the source of excitation is investigated. This problem had been solved via modal expansion technique. However, in case where the scatterer size is extremely large compared with the incident wavelength, the previously obtained solutions are no longer practical. In this paper, a practical treatment is developed. The Gaussian beam is expanded in terms of continuous plane waves with amplitudes and phases given by a Gaussian weighting functions. The continuous plane wave spectrum of the Gaussian beam is obtained. Since the scatterer size is very large compared with the incident wavelength, the scattering of each plane wave by the conducting circular cylinder is computed via geometrical optics technique. Thus, the evaluation of the Gaussian beam scattering from the conducting circular cylinder reduces to evaluating the inverse Fourier transform of the scattered plane waves. Numerical results using a code developed under MATLAB environment is obtained. The obtained solution is compared with the previously published solutions that are based on modal expansion technique and good agreement is achieved.

\section{KEYWORDS}

Laser Beam; Laser Scattering; Conducting circular cylinder; Coated cylinder; Concentric cylinders; Dielectric cylinder.

\section{INTRODUCTION}

The conducting cylinders represent one of the most important classes of geometrical surfaces. Many practical scatterers, such as fuselage of airplanes, pipelines, and missiles can be represented by cylindrical structures. The problem of scattering of an incident plane wave by a conducting cylinder had been extensively studied by many workers [7, 8, and21]. With the development of laser sources and the tremendous expansion of their application, attention is turned towards solving the scattering problem from a Gaussian beam rather than the plane wave. The scattering of a Gaussian beam by a conducting cylinder of the size comparable of the beam footprint had already also been studied by some workers [8-18].

Professor, Dean of NILES, Cairo University, Guiza, Egypt.

"Egyptian Armed Forces. 
Them large size conducting circular cylinder becomes the matter of interest for many researches and applications. The scattered field could be obtained using various techniques: modal techniques (MT) geometrical optics (GO), physical optics (PO), integral equations (IE), and diffraction theory such as the geometrical theory of diffraction (GTD) and physical theory of diffraction (PTD).

The modal expansion technique represents one of the major employed techniques. In this technique, the modal expansion of the incident waves is obtained. Then, applying the boundary condition for each constituent to get the modal expansion of cothe scatterer the scattered field. As the frequency the modal expansion of the scattered field becomes slow and one of the high frequency methods can be employed. On the other hand, the literature survey shows of the scattered waves due to Gaussian incident beam could be obtained via GO technique.

In this paper, a method for computation of the scattered field is developed to evaluate the Gaussian beam scattering from very large conducting cylinder. The evaluate the localization Gaussian beam is assun

The approach utilized in this paper is based on plane wave expansion of the incident Gaussian beam. The scattered fields due to each plane wave are calculated via The scattered field due to Gaussian beam excitation is obtained by summing via $\mathrm{GO}$. The scattered fue to each incident plane waves.

\section{PLANE WAVE EXPANSION OF THE INCIDENT GAUSSIAN BEAM}

The geometry of the problem is shown in Fig.1. An infinitely long perfectly conducting circular cylinder placed at the origin is excited by a TM laser beam located along the negative $z$-axis at a distance $z_{0}$ from the origin. Thus the beam width can be written as:

$$
w^{2}\left(z-z_{0}\right)=w_{n}^{2}\left[1+\left(\frac{\lambda\left(z-z_{0}\right)}{\pi w_{0}^{2}}\right)^{2}\right],
$$

where $\lambda$ is the free space wavelength and $w_{\mathrm{o}}$ is the radius of the beam at the waist ( at the exit of the source of lasing system ). The radius of curvature of the incident phase fronts is given by (2)

$$
R\left(z-z_{\mathrm{o}}\right)=z\left[1+\left(\frac{\pi w_{0}^{2}}{\lambda\left(z-z_{0}\right)}\right)^{2}\right] .
$$




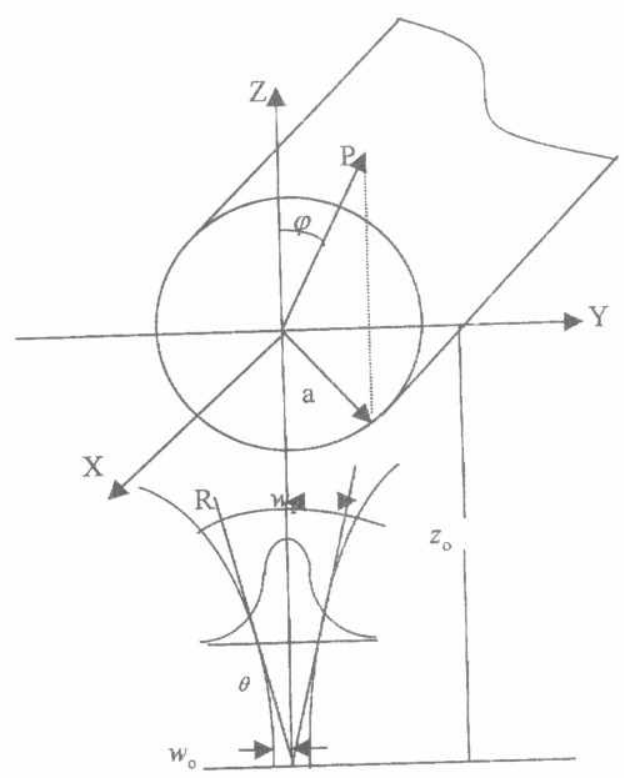

Fig. 1 Schematic diagram of the incident Gaussian beam on an infinitely long perfectly conducting circular cylinder of radius "a"

It is obvious from the equations that as the Gaussian beam propagates away from the location of its waist, the beam width (or spot size) at first remains nearly constant but then begins to diverge linearly with distance at large distances from the waist. the smaller the distance over which it stays collimated with a nearly constant diameter and a the angle $\theta$ in Fig. 1 is the beam divergence angle. It is given by (3).

$$
\theta=\frac{w\left(z-z_{0}\right)}{z-z_{0}}
$$

The source field is assumed to be represented by

$$
E_{x}^{i}\left(y,-z_{\mathrm{o}}\right)=E_{\mathrm{o}} e^{-\beta^{2} y^{2}}
$$

where, $\quad \beta^{2}=\frac{1}{w_{\mathrm{o}}^{2}} ; w_{\mathrm{o}}$ corresponds to the incident beam width 
The wave equation for the TM incident electric field component is given by:

$$
\frac{\partial^{2} E_{x}^{i}}{\partial x^{2}}+\frac{\partial^{2} E_{x}^{i}}{\partial y^{2}}+k_{\mathrm{o}}^{2} E_{x}^{i}=0 .
$$

where the wave number is defined by $k_{0}^{2}=\omega^{2} \mu_{0} \varepsilon_{0}, \omega$ is the angular frequency, $\mu_{0}$ and $\varepsilon_{0}$ are the permeability and permittivity of free space, respectively. The solution of (5) is accomplished by applying the continuous Fourier transform in the $y$ direction and then solving the transformed equation in closed form. Doing so, we obtain,

$$
E_{x}^{i}(y, z)=\frac{1}{2 \pi} \int_{-\infty}^{\infty} E_{i}(\alpha) e^{-i\left(z+z_{0}\right) \sqrt{k_{0}^{2}-\alpha^{2}}-i \alpha y} d \alpha .
$$

where the spectrum amplitude $E_{i}(\alpha)$ is represented by taking the Fourier transformation of the beam source as follows:

$$
E_{i}(\alpha)=\int_{-\infty}^{\infty} E_{x}^{i}\left(y,-z_{\mathrm{o}}\right) e^{r \alpha y} d y .
$$

Substituting by Eq.4 in Eq.7 we get

$$
E_{i}(\alpha)=\int_{-\infty}^{\infty} E_{0} e^{-\beta^{2} y^{2}} e^{i \alpha y} d y .
$$

Making some mathematical manipulation on the spectrum amplitude $E_{i}(\alpha)$ in $(8)$ and substituting in (6) one obtains

$$
E_{x}^{i}(y, z)=\frac{E_{0}}{2 \sqrt{\pi} \beta} \int_{-\infty}^{\infty} e^{-\frac{\alpha^{2}}{4 \beta^{2}}} e^{-i\left(z+z_{0}\right) \sqrt{k_{0}^{2}-\alpha^{2}}}{ }^{-i \alpha y} d \alpha .
$$

The above integral is the exact solution for computing the incident Gaussian beam that in terms of plane wave expansion.

\section{SOLUTION OF THE SCATTERING PROBLEM VIA MODAL EXPANSION}

To solve for the unknown field components, we apply the boundary conditions on the surface of the cylinder at $\rho=a$ in an iterative procedure. In this procedure, the incident Gaussian beam falls on the surface of the infinitely long circular cylinder and the scattered field has been occurred due to this interaction. 
The application of the boundary conditions on the surface of the conducting cylinder, are given by

$$
\begin{aligned}
& E_{t}=H_{n}=0 \text {. } \\
& E_{x}^{i}+E_{x}^{s}=0, \quad \rho=a, \quad 0 \leq \phi \leq 2 \pi . \\
& H_{x}^{i}+H_{x}^{s}=0, \quad \rho=a, \quad 0 \leq \phi \leq 2 \pi .
\end{aligned}
$$

where $E_{t}, H_{n}$ are the tangential component of the electric field and the normal component of the magnetic field respectively. $E_{x}^{i}$ is the incident Gaussian beam which is given by (4). In cylindrical coordinates (6) can be written as[8]

$$
E_{x}^{\prime}=E_{0} \sum_{n=-\infty}^{n=\infty} A_{n} j^{-n} J_{n}\left(k_{0} \rho\right) \cdot e^{j n \phi},
$$

where

$$
A_{n}=\frac{1}{2 \sqrt{\pi} \beta} \int_{-\infty}^{\infty} e^{-\frac{\alpha^{2}}{4 \beta^{2}}-j z_{0} \sqrt{k_{0}^{2}-\alpha^{2}}-j n \gamma(\alpha)} d \alpha,
$$

where,

$$
\gamma(\alpha)=\sin ^{-1}\left(\frac{\alpha}{k_{0}}\right)
$$

where $A_{n}$ represents the spectrum amplitude weighting function of the laser beam[17].

$E_{x}^{s}$ is the scattered field. Since the scattered field travels in the outward direction, they must be represented by cylindrical travelling wave function. Thus it can be represented by

$$
E_{x}^{s}=E_{0} \sum_{n=-\infty}^{n=\infty} c_{n} H_{n}^{(2)}\left(k_{0} \rho\right) e^{j n \phi} .
$$


where $c_{n}$ represents the unknown amplitude coefficient. This unknown coefficient can be found from the boundary condition.

Thus, by substituting of (13) \& (16) in (11) one obtains

$$
E_{0} \sum_{n=-\infty}^{n=\infty}\left[j^{-n} A_{n} J_{n}\left(k_{0} a\right)+c_{n} H_{n}^{(2)}(k 0 a)\right] e^{j n \phi}=0
$$

or

$$
c_{n}=-j^{-n} A_{n} \frac{J_{n}\left(k_{0} a\right)}{H_{n}^{(2)}\left(k_{0} a\right)} .
$$

By applying (18) in (16), the result is the cylindrical expansion of the scattered field due to the incident Gaussian beam that previously expanded in terms of plane waves.

$$
E_{x}^{s}=E_{0} \sum_{n=-\infty}^{n=\infty}-j^{-n} A_{n} \frac{J_{n}\left(k_{0} a\right)}{H_{n}^{(2)}\left(k_{0} a\right)} H_{n}^{(2)}\left(k_{0} \rho\right) e^{j n \phi}
$$

For plane wave incident the weighting function term $A_{n}$ will be tends to unity.

Also, the corresponding scattered magnetic field can be obtained by using Faraday's equation, which in these instances reduces to:

$$
\begin{aligned}
& H_{x}^{t}=-\frac{1}{i \omega \mu_{0}} \nabla \times E_{x}^{i} . \\
& H_{x}^{s}=-\frac{1}{i \omega \mu_{0}} \nabla \times E_{x}^{s} .
\end{aligned}
$$

Thus,

$$
\begin{aligned}
& H_{\phi}^{i}=-\frac{1}{i \omega \mu_{0}} \frac{1}{\rho} \frac{\partial E_{x}^{i}}{\partial \rho} . \\
& H_{\phi}^{\prime}=-\frac{1}{i \omega \mu_{0}} \frac{1}{\rho} E_{0} k_{0} \sum_{n=-\infty}^{\infty} i^{-n} A_{n} J_{n}^{\prime}\left(k_{0} \rho\right) e^{i n \phi} .
\end{aligned}
$$




$$
\begin{aligned}
& H_{\phi}^{s}=-\frac{1}{i \omega \mu_{\mathrm{o}}} \frac{1}{\rho} \frac{\partial E_{x}^{s}}{\partial \rho} . \\
& H_{\phi}^{s}=-\frac{1}{i \omega \mu_{\mathrm{o}}} \frac{1}{a} E_{\mathrm{o}} k_{\mathrm{o}} \sum_{n=-\infty}^{\infty} i^{-n} c_{n} H_{n}^{(2)^{\prime}}\left(k_{\mathrm{o}} \rho\right) e^{i n \phi} .
\end{aligned}
$$

\section{FAR-ZONE SCATTERED FIELD}

One of the most important parameters in scattering is the scattering width which is obtained by knowing the scattered field in the far zone. For this problem this can be accomplished by first reducing the scattered fields for far-zone observation ( $k_{0} a \rightarrow$ large). The Hankel function can be approximated in the far field by

$$
\lim _{k_{0} \rho \rightarrow \infty} H_{n}^{(2)}\left(k_{0} \rho\right) \approx \sqrt{\frac{2 j}{\pi k_{0} a}} j^{n} e^{-j k_{0} \rho}
$$

When substituting by Eq. 26 in Eq.19, we get

$$
E_{x}^{s} \stackrel{k_{0} \rho \rightarrow \infty}{\approx}-E_{0} \sqrt{\frac{2 j}{\pi k_{0}}} \frac{e^{-j k_{0} \rho}}{\sqrt{\rho}} \sum_{n=-\infty}^{n=\infty} \frac{J_{n}\left(k_{0} a\right)}{H_{n}^{(2)}\left(k_{0} a\right)} e^{j n \phi}
$$

The ratio of the far- zone scattered electric field to the incident field can then be written as[8]:

$$
\begin{aligned}
\frac{\left|E_{x}^{s}\right| k_{0} \rho \rightarrow \text { arge }}{\left|E_{x}^{i}\right|} \stackrel{\left|-E_{0} \sqrt{\frac{2 j}{\pi k_{0}}} \frac{e^{-j k_{0} \rho}}{\sqrt{\rho}} \sum_{n=-\infty}^{n=\infty} A_{n} \frac{J_{n}\left(k_{0} a\right)}{H_{n}^{(2)}\left(k_{0} a\right)} e^{j n \phi}\right|}{\left|E_{0} \sum_{n=-\infty}^{n=\infty} A_{n} j^{-n} J_{n}\left(k_{0} \rho\right) \cdot e^{j n \phi}\right|} . \\
=\sqrt{\frac{2}{\pi k_{0} \rho}\left|\sum_{n=-\infty}^{n=\infty} \frac{J_{n}\left(k_{0} a\right)}{H_{n}^{(2)}\left(k_{0} a\right)} e^{j n \phi}\right| .}
\end{aligned}
$$


Thus the scattering width (SW)can be expressed as[8]:

$$
\begin{aligned}
\mathrm{SW}=\sigma_{2-D} & =\lim _{\rho \rightarrow \infty}\left[2 \pi \rho \frac{\left|E_{x}^{s}\right|^{2}}{\left|E_{x}^{t}\right|^{2}}\right]=\frac{4}{k_{0}}\left|\sum_{n=-\infty}^{n=\infty} \frac{J_{n}\left(k_{0} a\right)}{H_{n}^{(2)}\left(k_{0} a\right)} e^{j n \phi}\right|^{2} . \\
& =\frac{2 \lambda}{\pi}\left|\sum_{n=0}^{n=\infty} \frac{J_{n}\left(k_{0} a\right)}{H_{n}^{(2)}\left(k_{0} a\right)} \cos (n \phi)\right|^{2} .
\end{aligned}
$$

\section{COMPUTATION OF SCATTERING FIELD USING GEOMETRICAL OPTICS THEORY}

The laws of geometrical optics are asymptotic laws on electromagnetic waves, valid in the limit of very small wavelength relative to the scatterer size. In this work, the scatterer size is very large compared with the incident wavelength. The scattering of each plane wave by the conducting circular cylinder is computed via geometrical optics technique, as shown in fig.2, to overcome the slow convergence encountered when the computation has been done using modal expansion technique.

The formulae for cylindrical objects, can be obtained as[21]

$$
E_{s}=S(\varphi) \cdot \frac{e^{-i k r+i k z}}{i k r} \cdot E_{i}
$$

where $E_{s}$ the scattered electric field, $E_{i}$ is the incident electric field, $\varphi$ is the scattering angle, and $\mathrm{S}(\varphi)$ the complex amplitude function describing the amplitude and phase of the scattered field.

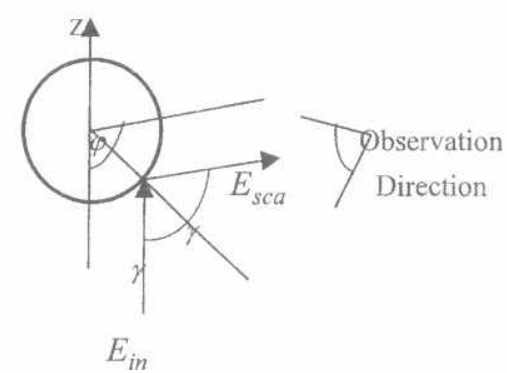

Fig.2. Geometrical optics technique 
The complex amplitude function is defined as [21]

$$
S(\varphi)=\frac{1}{2} i x e^{2 i x \sin \left(\frac{\varphi-\gamma}{2}\right)} .
$$

where $\mathbf{X}$ is the size parameter, $\varphi$ is the scattering angle, and $\gamma$ is the incident angle.

By applying Eq.9, Eq.15, and Eq.33 in Eq.32 the scattered field is given as

$$
E_{s}=\frac{E_{0} a}{4 \beta \sqrt{\pi}} \cdot \frac{e^{-i k_{\delta}}}{r} \cdot \int_{-\infty}^{\infty} e^{-\frac{\alpha^{2}}{4 \beta^{2}}-i\left(z_{0} \sqrt{k_{0}^{2}-\alpha^{2}}-2 x \sin \left(\frac{\varphi-\gamma}{2}\right)\right)} d \alpha .
$$

\section{NUMERICAL RESULTS}

The equations derived in the previous section for the scattered field are solved numerically. The integration over $\alpha$ that runs from $-\infty$ to $\infty$ is truncated to a very high values $\alpha_{T}$ that depends on the incident beam parameters and the scatterer size. $\alpha_{T}$ is chosen such that further increase in its value will not affect the integration value.

Computer codes written with MATLAB environment are developed to solve these equations. Different cases that correspond to different beam parameters and scatterer size are considered. In all case the scattered width (SW) that defined by Eq. 30 is computed.

The scattered width versus scattering angle is computed for certain scatterer size parameter $\mathrm{X}$ with several spot size to radius ratios $\left(W_{z} / a\right)$ that given by $0.2,1$, and 3 as shown in Figs. $5-7$. The comparison between the computation using both of modal expansion solution and asymptotic solution (GO) has been given as shown in Figs. $8-10$.

Fig. 3, for code verification, shows a good agreement between the obtained results from our code (dashed curve), in case of incident plane wave, and the previously published results in [8] (solid curve).

Fig. 4 shows a comparison between the scattering width in case of incident plane wave and the scattering width in case of incident Gaussian beam with different spot size to radius ratio.

Figs. $5-7$ show the scattering width of the cylinder for various beam spot size to radius ratio given by $W_{z} / a=0.2,1$, and 3 at certain scatterer size parameter that is given by $X=10,50$, and 100 respectively. 
In view of the previous figures, it is clear that as the footprint size of the incident Gaussian beam increases the scattering pattern approaches that due to incident plane waves.

On the other hand, decreasing the spot size relative to the scatterer dimensions results the induction current localization in the considered cases $(X>>1)$ in the illuminated region only and thus, the scattering is confined in the backward side. However, when the beam footprint covers the whole cylinder, induced currents are created in the shadow region and give rise to scattering in the forward half side.

Figs. $8-10$, show a comparison between the computation of scattering width by using the exact solution (modal expansion) and asymptotic solution by using the $\mathrm{GO}$ technique. The computation has been given for two beam spot size to radius ratio given by $W_{2} / a=0.2$, and 3 at certain scatterer size parameter $\mathrm{X}$ that is given by $X=10,50$, and 100 respectively.

The obtained results indicate that the accuracy of the asymptotic solution increases as the size parameter $X$ increases which is an expected result.

In the mean time, the footprint relative to spot size ratio does not affect the accuracy of the asymptotic solution. Thus the asymptotic solution can be adopted in the computation of the scattered field from the incident Gaussian beam, since the cornputation time is highly reduced.

Table 1 shows the computation time of the scattered width via the exact and the asymptotic methods, on a Pentium II- $450 \mathrm{HHz}$ IBM compatible PC. It is obvious from the table that the computation time via modal expansion technique increases by increasing the size parameter $\mathrm{X}$. However by employing the $\mathrm{GO}$ approximation, the computation time is highly reduced. This is considered the major advantage of adopting the GO asymptotic solution especially, when considering scattering from very large objects where the size parameter $\mathrm{X}$ is few millions (as in typical LIDAR systems).

Table 1. The execution times in seconds used for the computation of the scattering radiation patterns that computed by modal expansion and geometrical optics techniques on IBM compatible PC Pentium II-450MHZ.

\begin{tabular}{|c|c|c|c|}
\hline \multirow{2}{*}{$\begin{array}{c}\text { Scattering } \\
\text { Width }[\mathrm{SW}]\end{array}$} & Size & \multicolumn{2}{|c|}{$\begin{array}{c}\text { Gaussian } \\
\text { Execution }\end{array}$} \\
\cline { 3 - 4 } Pattern in & $\begin{array}{c}\text { Beam } \\
\text { time in sec. }\end{array}$ \\
\cline { 3 - 4 } & {$[\mathrm{X}]$} & $\begin{array}{c}\text { Modal } \\
\text { Expansion }\end{array}$ & $\begin{array}{c}\text { Geometrical } \\
\text { Optics }\end{array}$ \\
\hline Fig. 6 & 10 & 27 & 2 \\
\hline Fig. 7 & 50 & 118 & 3 \\
\hline Fig.8 & 100 & 230 & 5 \\
\hline
\end{tabular}




\section{CONCLUSION}

1- The scattering pattern from conducting circular cylinder due to incident Gaussian beam of spot size to cylinder radius ratio given by $\left(W_{z} / a>1\right)$ approaches that due to incident plane wave.

2- The scattering pattern from conducting circular cylinder due to incident Gaussian beam of spot size to cylinder radius ratio given by $\left(W_{z} / a<1\right)$ becomes more confined in the backward direction.

3- The utilization of the asymptotic solution using the GO technique allows the fast computation of Gaussian beam scattering from extremely large scatterer with size parameter $\mathrm{X}>>1$.

\section{REFERENCES}

1- Abramwitz, M., and I. Stegun, (1965), Handbook of Mathematical Functions, New York : Dover.

2- A. Ungut, G. Gerhan, and G. Gouesbet, (1981), Comparisons between geometrical optics and Lorenz-Mie theory, Applied Optics, Vol. 20, No. 17, P.P. 2911- 2918.

3- A. M. Azzam et al, (2000), Gaussian beam scattering from very large conducting sphere with size comparable with beam footprint, Al-azhar Sixth International Engineering Conference, AIEC, Cairo, Egypt, PP. 266-282

4- A. M. Azzam et al, (2001), Laser beam scattering from metallic sphere embedded in aerosol cloud, Eighteenth National Radio Science Conference, March 27-29, Mansoura, Egypt.

5- Antonio Collaro and et al., (1999), Gaussian rough surfaces and Kirchhoff approximation, IEEE Trans. Antennas Propagat., Vol. 47, No. 2, PP. 392-397.

6- A. Z. Elsherbeni and et al., (1993), Iterative scattering of a Gaussian beam by an array of circular conducting and dielectric cylinders, Journal of Electromagnetic waves and applications, Vol. 7, No. 10, P.P $1323-1342$.

7- A. Z. Elsherbeni and A. Kishk, (1992), Modeling of cylindrical objects by circular dielectric or conducting cylinders, IEEE Trans. Antennas Propagat., Vol. AP-40, P.P 96-99.

8- Balanis,C.A., (1989), Advanced Engineering Electromagnetics, New York Wiely.

9- Harrington, R.F., (1968), Field Computation By Moment Methods, MacMillan, New York.

10- James A. Lock, (1995), Improved Gaussian beam-scattering algorithm," Applied Optics, Vol. 34, No. 3, P.P 559-570. 
11- James A. Lock, (1995), Interpretation of extinction in Gaussian-beam scattering, J. Opt. Soc. Am. A., Vol. 12, No. 5, P.P 929-938

12- K. F. Ren, G. Grehan, and G. Gouesbet, (1997), Scattering of a Gaussian beam by an infinite cylinder in the framework of generalized Lorenz-Mie theory: formulation and numerical results, J. Opt. Soc. Am. A., Vol. 14, No. 11, P.P 3014 3025 .

13- Langlois and et al., (1985), Electromagnetic diffraction of a transverse twodimensional Gaussian beam at normal incidence on an absorbing circular cylinder, Can. J. Phys., Vol. 63, P.P $301-309$.

14- L. C. Andrews, (1993), New results concerning Gaussian beam wave propagation," SPIE Vol. 1968 Atmospheric propagation and remote sensing II P.P 204-216.

15- N. G. Alexopoulos and P. K. Park, (1972), Scattering of waves with normal amplitude distribution from cylinders, IEEE Trans. Antennas Propagat., AP-20, P.P. 216-217.

16- S. Choudhary and L. B. Felsen, (1974), Analysis of Gaussian beam propagation and diffraction by inhomogeneous wave tracking, IEEE Trans. Antennas Propagat., Vol. 62, No. 11, P.P. 1530-1541

17- Shogo Kozaki, (1982), A new expression for the scattering of a Gaussian beam by a conducting cylinder, IEEE Trans. Antennas Propagat., Vol. AP-30, P.P 881-887.

18- Shogo Kozaki, (1982), Scattering of a Gaussian beam by an inhomogeneous dielectric cylinder, J. Opt. Soc. Am., Vol. 72, No. 11, P.P 1470-1474.

19- Shogo Kozaki and H. Sakurai, (1978), Characteristics of a Gaussian beam at a dielectric interface, J. Opt. Soc. Am., Vol. 68, No. 4, P.P 508-514.

20- T. Kojima and Y. Yanagiuchi, (1978), On the scattering of an offset twodimensional Gayssian beam wave by a cylinder, Electron. Commun. (Japan) J61B, P.P $472-478$.

21- Van de Hulst, (1981), Light scattering by small particles, Dover, New York. 


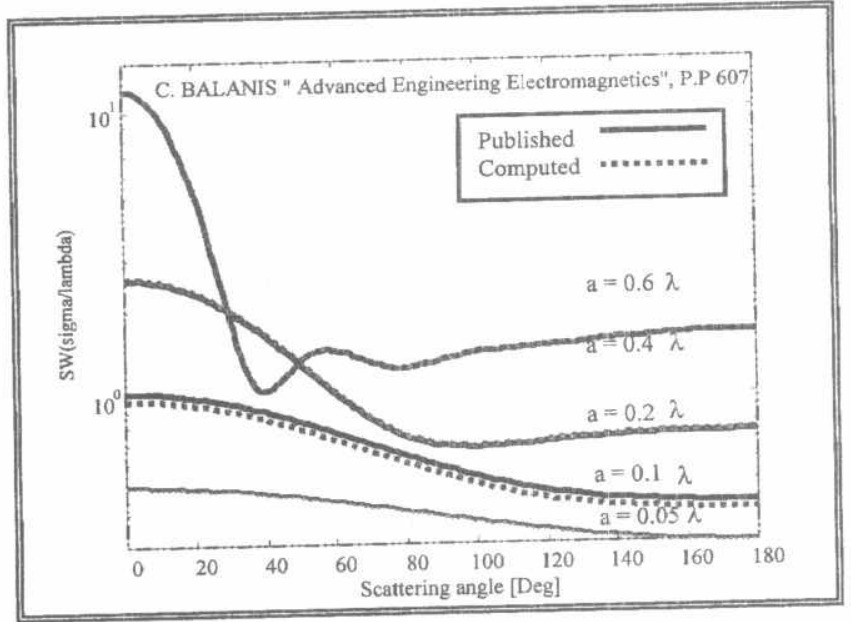

Fig.3.Two dimension scattering width of a circular conducting cylinder in case of plane wave incident

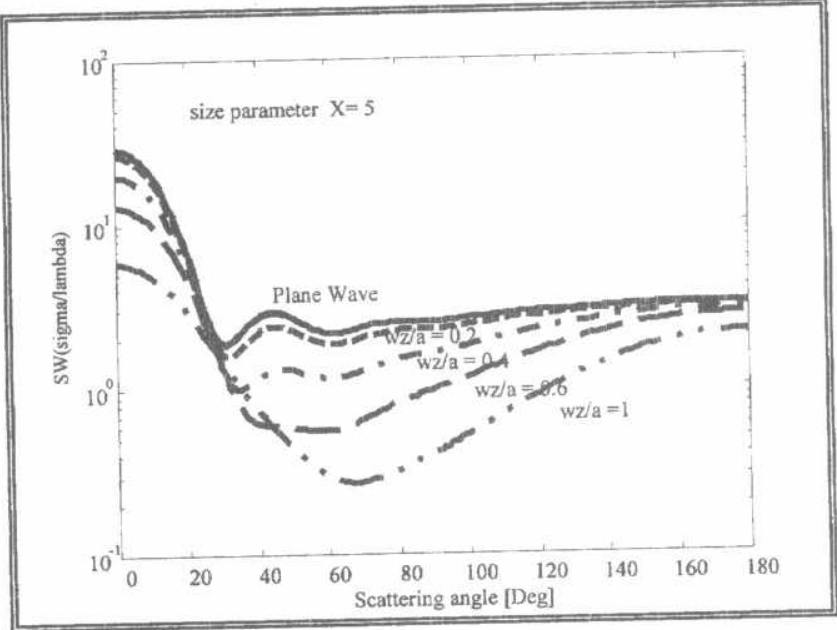

Fig.4.Two dimension scattering width of a cicular conducting cylinder in case of Gaussian beam incident $(X=5)$. 


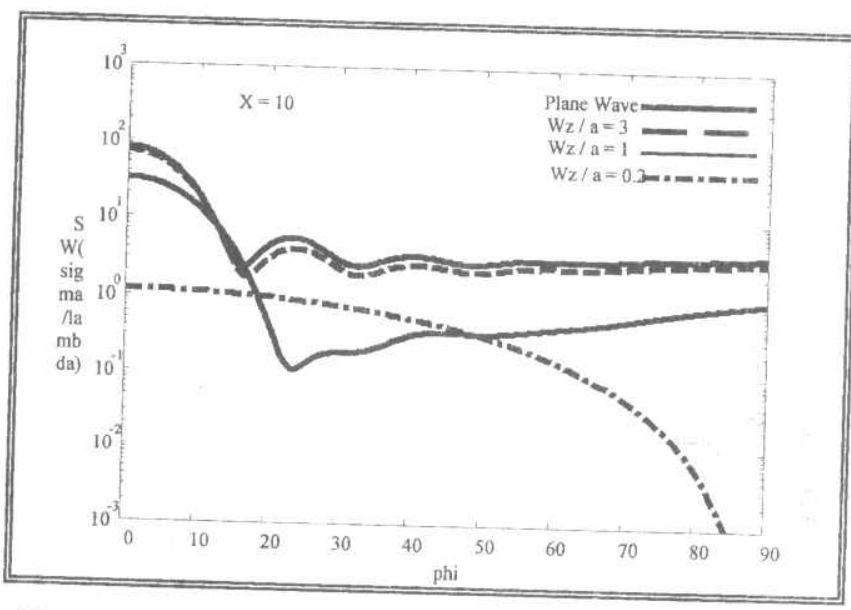

Fig. 5 Scattering width for a conducting circular cylinder of size parameter $X=10$ in case of plane wave and Gaussian beam incident with different spot sizes as a function of their radii

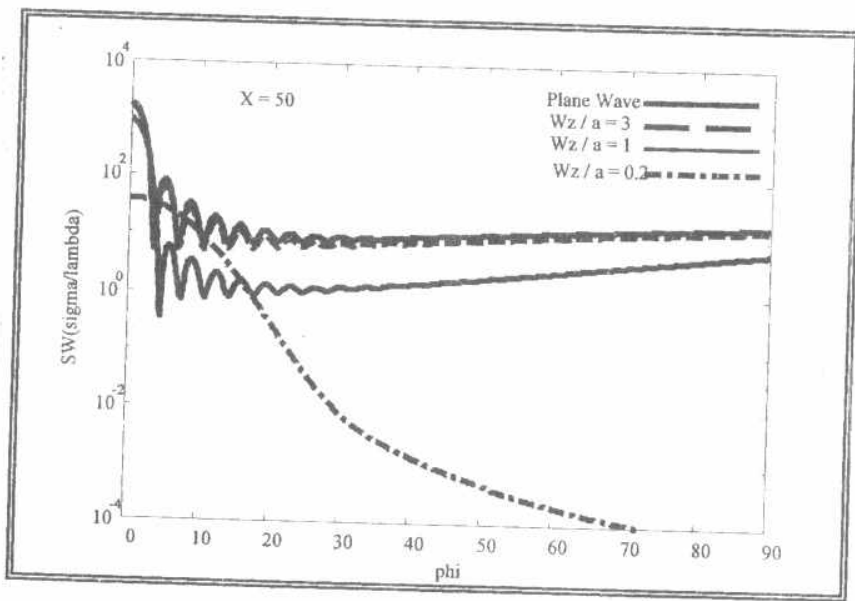

Fig. 6 Scattering width for a conducting circular cylinder of size parameter $X=50$ in case of plane wave and Gaussian beam incident with different spot sizes as a function of their radii 


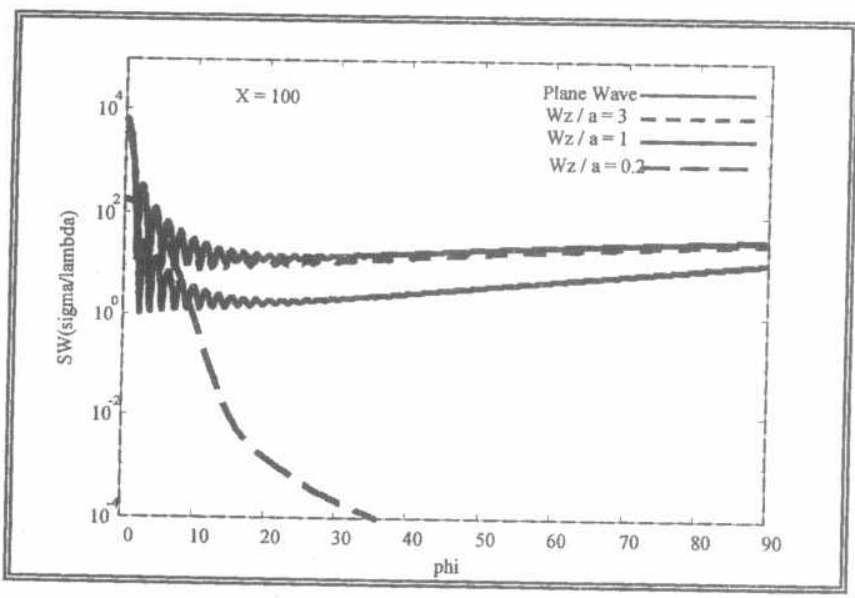

Fig. 7 Scattering width for a conducting circular cylinder of size parameter $X=100$ in case of plane wave and Gaussian beam incident with different spot sizes as a function of their radii

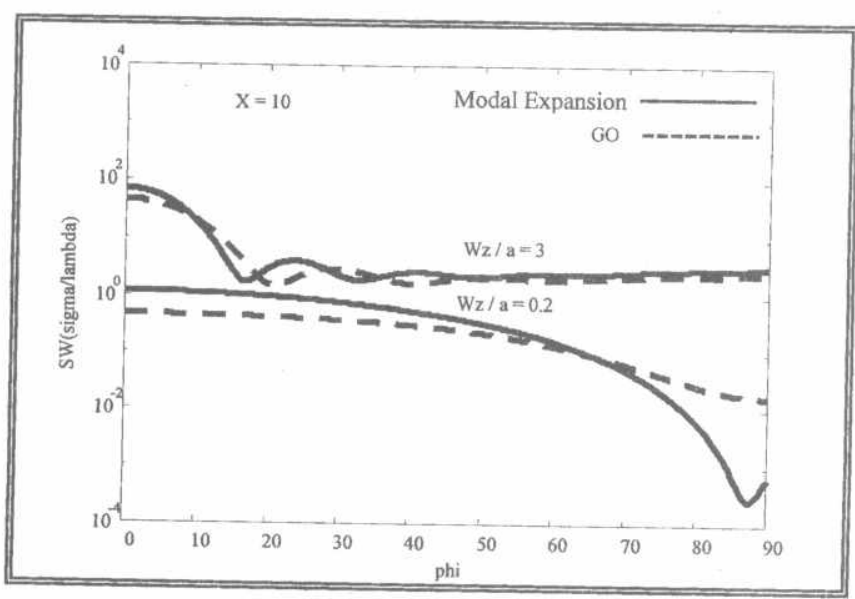

Fig. 8. Scattering width of Gaussian beam incident on a conducting circular cylinder of $X=10$ and spot size to radius ratio $\mathrm{Wz} / \mathrm{a}=1$ by using modal expansion and $\mathrm{GO}$ 


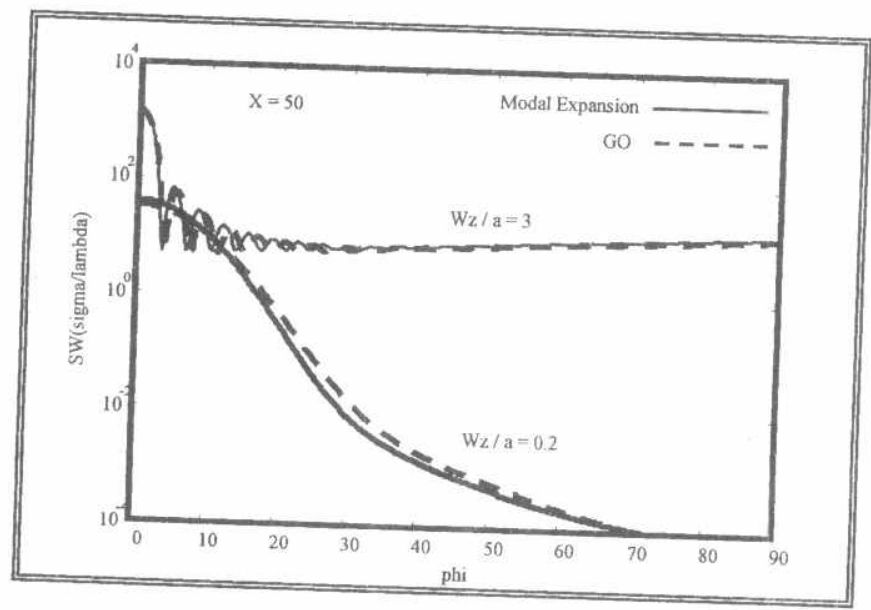

Fig. 9 Scattering width of Gaussian beam incident on a conducting circular cylinder of $X=50$ and spot size to radius ratio $\mathrm{Wz} / \mathrm{a}=1$ by using modal expansion and $\mathrm{GO}$

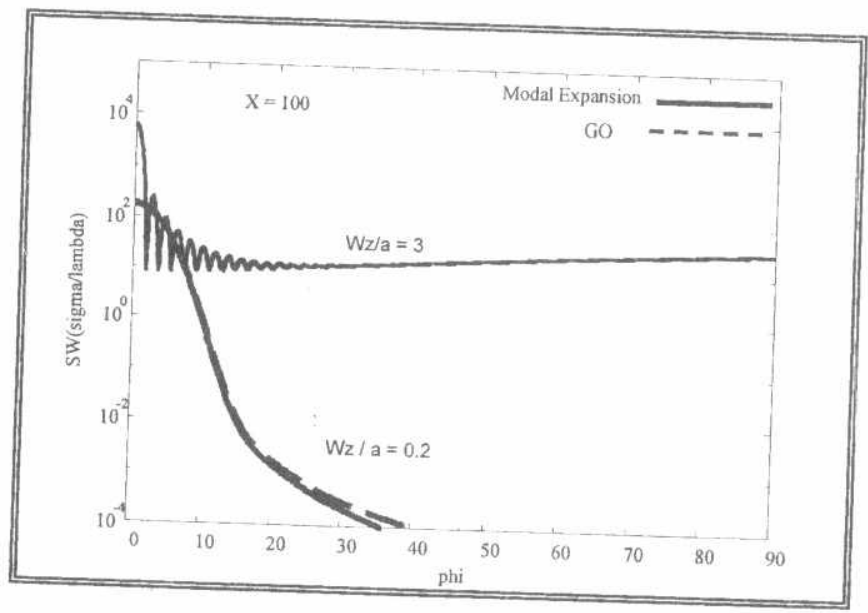

Fig. 10 Scattering width of Gaussian beam incident on a conducting circular cylinder of $X=100$ and spot size to radius ratio $\mathrm{Wz} / \mathrm{a}=1$ by using modal expansion and $\mathrm{GO}$ 